\title{
New Aboriginal health course increases awareness
}

A research project by 2 McMaster University Medical School students blossomed into a 5 month course this year, focussing on Aboriginal health. Thirty students signed on for the elective course, the first of its kind in Canada, which offered lectures and instruction from both Aboriginal and non-Aboriginal speakers as well as problembased learning, clinical place$\infty$ ments and community visits.

Todd Young, a third-year medical student who worked as ह nurse practitioner in Northồ ern Ontario Aboriginal communities for 10 years before study-

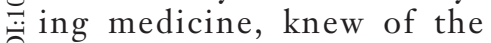
$\AA$ difficulties faced by non-Abo- riginal physicians treating Aboriginal patients.

"Part of medical education is to develop a culturally-sensitive, diverse health care provider, sensitive to the needs of Aboriginal people," Young said. "To do this, you have to be aware of the issues. A person growing up in Toronto may not have a fair assessment of the issues that affect the health of Aboriginal people."

Dr. Cornelia Wieman, director of McMaster's Native Students Health Sciences office and one of only 150 Aboriginal physicians in Canada, helped to develop the elective. "The focus of the elective is on how the medical students can participate in contributing to the improved health status of Aboriginal peoples by delivering more culturally relevant care."

Statistics Canada reported in its 2001 census that the total Aboriginal population in Canada was 976305.

"When we look at realities, most Aboriginal people access health care from non-Aboriginal physicians," Young said.

The decision on whether to offer the course again is still being examined, but Young said that "the student feedback has been overwhelming. They learned about issues they didn't even know about before." Ken Kilpatrick, Hamilton

Pulse

\section{Younger physicians, specialists use Internet more}

Sixty-six percent of Canadian physicians in clinical practice use the Internet as a tool or source of information to support treatment or in direct patient care, according to the CMA's 2003 Physician Resource Questionnaire (PRQ).

$\vec{\sim}$ Searching online medical data-

bases is the most popular use of the Internet among clinicians (reported by $57 \%$ ), followed by referring to online clinical practice guidelines (41\%) and reading online medical journals $\stackrel{\circ}{\circ}(38 \%)$. Sixteen percent of physicians in clinical practice refer to online drug databases, while $8 \%$ receive secure patient-specific information over the Internet and 6\% visit other physicians' Web sites.

Younger physicians are more likely to access some types of einformation than their older colleagues. Seventy-three percent of those under age 35 and $63 \%$ in the 35-44 age range report that they search online medical databases, compared to half of clinicians aged $55-64$ and $39 \%$ of those 65 and older. Similarly,

Percentage of Canadian physicians using Internet tools in clinical practice

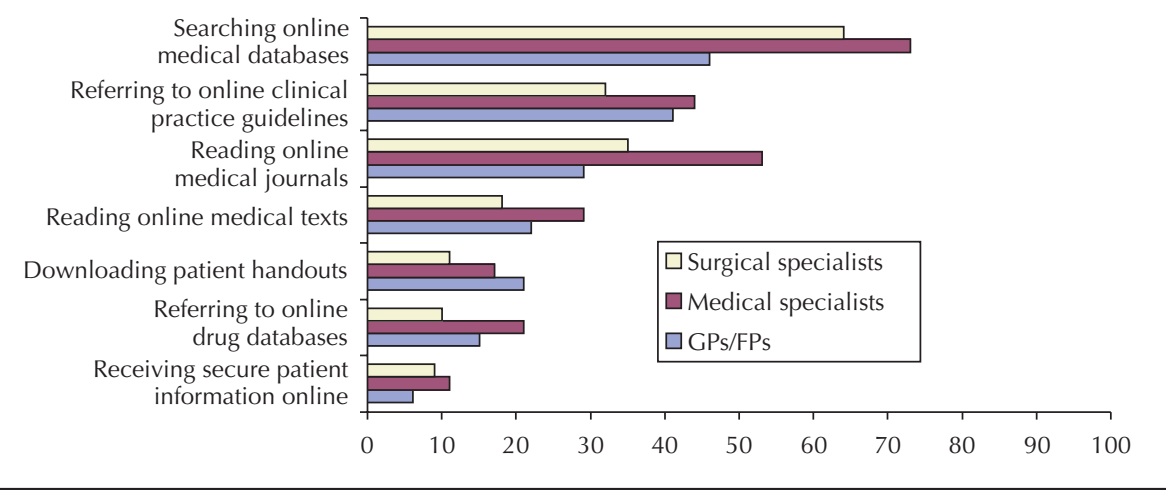

$61 \%$ of clinicians under 35 and $48 \%$ aged $35-44$ refer to online CPGs, compared to $23 \%$ of physicians aged 65 or more. Agerelated differences persist for reading online medical journals and textbooks, but are not apparent for reading online drug databases, receiving secure patientspecific information, and visiting other physicians' Web sites.

Medical specialists are more likely $(73 \%)$ to search online medical databases than surgical specialists $(64 \%)$ or FPs (43\%), and a similar pattern is evident for the reading of online medical journals and medical texts. Surgical specialists are less likely $(32 \%)$ to refer to online CPGs than medical specialists (44\%) or FPs (41\%).

The PRQ is Canada's largest annual survey of the professional activities of physicians. The 2003 survey was mailed to a random sample of 7922 doctors, and the response rate was $28.4 \%$. Results at the national level are considered accurate to within $\pm 2.1 \%$, 19 times out of 20. - Shelley Martin, Senior Analyst, CMA Research, Policy and Planning 